\title{
An Object Recognition and Identification System Using the Hough Transform Method
}

\author{
Jaruwan Toontham and Chaiyapon Thongchaisuratkrul
}

\begin{abstract}
This paper presents an object recognition and identification system using the Hough Transform method. The process starts from imported images into the system by webcam, detected image edge by fuzzy, recognized the object by Hough Transform, and separated the objects by the robot arm. Three objects type; triangle, rectangular and, rigid circle are used. The results showed that the objects can be isolated $96 \%, 96 \%$, and $98 \%$ correct for triangular, rectangular, and rigid circle respectively.
\end{abstract}

Index Terms-Hough transform, modifier fuzzy sobel, object recognition

\section{INTRODUCTION}

Nowadays, the robots are used widely in many applications such as automotive industry, rescue and security areas, medical exploration robotic, etc. Manipulator arms are employed in the industrial as the human arm for welding, lifting, separating, etc. Sometimes referred to as robot arm also means that the robot in industry. The robots will be played greater roles in the industry as much as the robot control algorithms are developed. They will work in various hazardous jobs such as lifting steel into furnace, job-related chemicals, repetitive monotonous work such as lifting or packing the objects in production line, desired quality such as welding and cutting, or the jobs that required highly skills such as welding line, welding laser, etc.

In this research, we are presented an object recognition and identification system using the Hough Transform method. There are many researches that used the Hough Transform method. Basak and Pal generalize the classical Hough Transform in fuzzy set theoretic framework in order to handle the imprecise in shape description called fuzzy Hough transform [1]. Maji and Malik present a discriminative Hough transform based object detector where each local part casts a weighted vote for the possible locations of the object center. The weights can be learned in a max-margin framework which directly optimizes the classification performance and improved the Hough detector [2]. Rizon and others use the circular Hough transform to detect the presence of circular shape [3]. Smereka and Duleba used The Hough Transform to improve the detection of low-contrast circular objects [4]. The Hough Transform can be implemented in C as discussed by Lee [5]. It also was modify to reduce memory area, computational time, and detect noise which was present by Sirisantisamrid and et al [6].

Manuscript received April 24, 2012; revised June 20, 2012.

The authors are with with King Mongkut's University of Technology North Bangkok, Bangkok,Thailand (e-mail: nudiddl@hotmail.com, srp@kmutnb.ac.th)
Khoshelham used laser range data and modified the generalized Hough Transform to detect 3D objects [7]. Alshennawy and Aly introduce the fuzzy logic reasoning to detect the digital images edge [8]. The linear Sobel operator was used to give a permanent effect in the lines smoothness and straightness for the straight lines and good roundness for the curved lines.

In our work, a robot arm was designed to use a fuzzy logic to detect the image edge [8] and to use Hough Transform method to recognize, identify, and separate the objects. The triangle, rectangular, and rigid circle objects are used to verify the system.

\section{IMAGE PREPROCESSING AND ENHANCE}

The images of the object that used in our experimental have different quality such as brightness, contrast, or noise. The fuzzy image processing (FIP) is used to adjust brightness of the image [8]. The process includes image fuzzification, membership modification, and image defuzzification which can be shown in Figure 1.

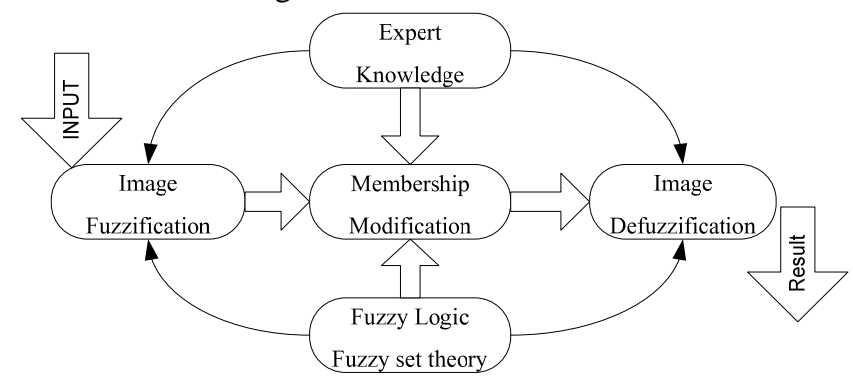

Fig. 1. Fuzzy image processing

To adjust the brightness of the image using the INT operator with the following methods are used. The original image matrix $\mathrm{X}$ of $\mathrm{N} \mathrm{x} \mathrm{M}$ is the array of fuzzy singleton of each member represented by the degree of brightness levels $p, p=0,1,2,3 \ldots P-1$ with $\mathrm{p}$ values between 0 and 255 can be written as follows.

$$
X=\left(\begin{array}{llll}
\mu_{11} / x_{11} & \mu_{12} / x_{12} & \ldots & \mu_{1 M} / x_{1 M} \\
\mu_{21} / x_{21} & \mu_{22} / x_{22} & \ldots & \mu_{2 M} / x_{2 M} \\
: & & & \\
\mu_{N 1} / x_{N 1} & \mu_{N 2} / x_{N 2} & \ldots & \mu_{N M} / x_{N M}
\end{array}\right)
$$

where $0 \leq \mu_{m n} \leq 1, m=1,2 \ldots M, n=1,2 \ldots N$ Using equation (1) to calculated.

$$
\begin{aligned}
T_{1}\left(\mu_{m n}\right) & =T_{1}^{\prime}\left(\mu_{m n}\right)=2 \mu_{m n}{ }^{2}, & & 0 \leq \mu_{m n} \leq 0.5 \\
& =T_{1}^{\prime \prime}\left(\mu_{m n}\right)=1-2\left(1-\mu_{m n}\right)^{2}, & & 0.5 \leq \mu_{m n} \leq 1
\end{aligned}
$$


Adjusted each the Pixel of image brightness as follow, values greater than 0.5 increases the brightness of pixels, values less than 0.5 decreases the brightness_of pixels. The procedure of the fuzzy image enhancement is shown in Fig. 2.

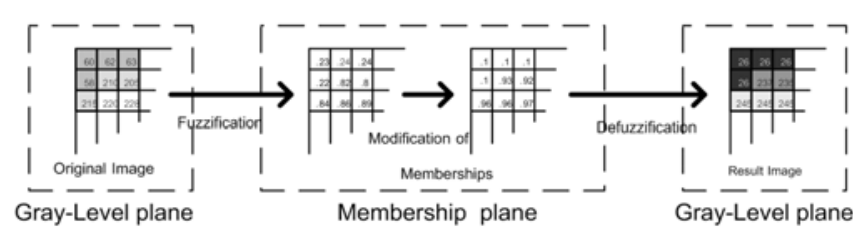

Fig. 2. Fuzzy Image Enhancements

\section{EAse of Use EdGe Detection Using Modifier FuZZY SOBEL $[10,11]$}

The modifier fuzzy Sobel is used for the edge detection. The steps are described as follows.

\section{A. Change the Image Color}

The image colors are changed to gray scale.

\section{B. Image Fuzzy Region}

Divide the image of Fuzzy regions into two parts: fuzzy smooth region and fuzzy edge region. If the pixels have difference values of gray scale level higher than the neighboring pixels, the result is a fuzzy edge region. If the pixels have difference values of gray scale level less than the neighboring pixels, the result is a fuzzy smooth region. The images edge of two parts has 4 value of the threshold to make a decision. The threshold value is derived from the calculation of the difference of the image histogram.

\section{Make Difference Histogram}

The difference histogram was built from the images by calculating the difference highest-level grayscale of all other pixels and 8 pixels neighboring of the surrounding as shown in Fig. 3. The ranges from 0 to $2^{\mathrm{m}-1}$ where $\mathrm{m}$ is number of bit of image gray level. In the case, represented of image by 8-bit is between 0 and 255 .

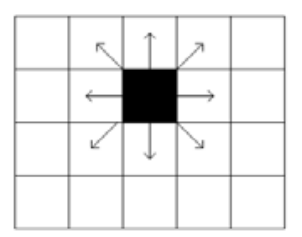

Fig. 3. The difference value of the pixel surrounding

\section{Find Difference Histogram}

The difference histogram is needed to find by using the formula $d(x, y)=\max (I(x, y)-I(x+i, y+j)$. When $\mathrm{I}(\mathrm{x}, \mathrm{y})$ is value of gray level of the input image at $(x, y), d$ is the most different values at $(\mathrm{x}, \mathrm{y})$ close to 8 pixels surrounding, and $\mathrm{I}(\mathrm{x}$ $+i, y+j)$ surrounding with $I(x, y)$ when $i=-1,0,1$ and $j=-1$, 0,1 .

\section{E. Set Threshold Values Determination}

The threshold value consists of 4 characters, LT $=$ Low Threshold, HL = High Limit, LL = Low Limit, and HT = High Threshold. To finding the edge of the image, the image mapping and the fuzzy regions are obtained as shown in Fig. 4 and 5.

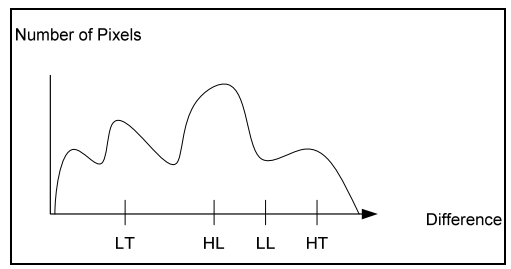

Fig. 4. Mapping Threshold 4 position

The threshold values determination is divided in to four equally ranges. If LT and HT can find, thus HL and LL was able to find as well.

\section{F. Fuzzy Region}

From four values of the threshold, two the fuzzy sets can be defined; fuzzy smooth region (SF) and fuzzy edge region (EF) as shown in Fig. 5.

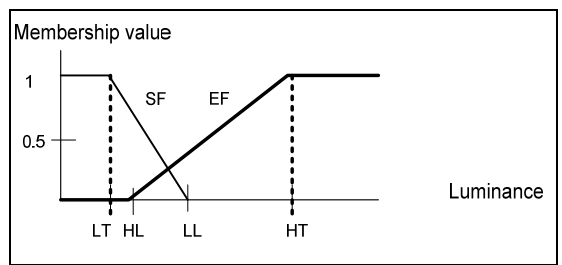

Fig. 5. Image fuzzy region obtained from the 4 Threshold values.

G. Find the Images Edge by Sobel

Identified the images edge by using two templates sizes $3 \times 3$.The first template for the difference in the horizontal ( $X_{\text {diff }}$ and the second template for the difference in vertical $\left(Y_{\text {diff }}\right)$ as shown in Fig. 6.

$X_{\text {diff }}=\left[\begin{array}{rrr}-1 & 0 & 1 \\ -2 & 0 & 2 \\ -1 & 0 & 1\end{array}\right] \quad Y_{\text {diff }}\left[\begin{array}{rrr}1 & 2 & 3 \\ 0 & 0 & 0 \\ -1 & -2 & -1\end{array}\right]$

Fig. 6. Templates of sobel

Input image can be calculated by the Sobel method equation (2)-(5)

$$
\begin{gathered}
\nabla f=\left[\begin{array}{l}
G x \\
G y
\end{array}\right]\left[\begin{array}{l}
\partial f \\
\partial x \\
\partial f \\
\partial y
\end{array}\right] \\
S(x, y)=\tan ^{-1}\left(\frac{G y}{G x}\right) \\
G_{x}=\left(Z_{7}+2 Z_{8}+Z_{9}\right)-\left(Z_{1}+2 Z_{2}+Z_{3}\right) \\
G_{y}=\left(Z_{3}+2 Z_{6}+Z_{9}\right)-\left(Z_{1}+2 Z_{4}+Z_{7}\right)
\end{gathered}
$$

\section{H. Fuzzy Reasoning for Edge Detection}

Fuzzy reasoning for edge detection is the result of algorithms modified fuzzy method to find the pixel position $(\mathrm{x}, \mathrm{y}), \mathrm{R}(\mathrm{x}, \mathrm{y})$ given by equation (6)

$$
R(x, Y)=\left\{\begin{array}{lr}
255 & S(x, y) \geq H T \\
0 & S(x, y) \leq L T \\
S(x, y) * \max \left(\mu_{S F}(x, y), \mu_{E F}(x, y)\right) & \text { otherwise }
\end{array}\right.
$$


When $\mathrm{S}(\mathrm{x}, \mathrm{y})$ is the value derived from the Sobel operator, $\mathrm{R}(\mathrm{x}, \mathrm{y})$ is the result of the pixel at $(\mathrm{x}, \mathrm{y})$, and $\mu_{\mathrm{SF}}, \mu_{\mathrm{EF}}$ are the membership functions of SF and EF. When Sobel gradient $\mathrm{S}(\mathrm{x}, \mathrm{y})$ was less than or equal to LT the result is 0 . If Sobel gradient $\mathrm{S}(\mathrm{x}, \mathrm{y})$ was more than or equal to HT the result is 255. If the result is not in either case, check the condition and then repeat to get results.

\section{Conventional Hough Transform (CHT)}

The conventional Hough transform is used to shape recognition by detecting linear curve and circle in the picture. For detection as linear the equation (7) is given [2]-[4].

$$
\rho=x \cos \theta+y \sin \theta
$$

When $x, y$ coordinate of the pixel on the image plane $x, y$, $\rho$ is the perpendicular distance from the origin to straight lines in plane image $x, y$, then the $\theta$ is the angle from $x$-axis perpendicular.

Two-dimensional accumulator cell A(i, j) was created which components of $\rho_{\mathrm{i}}, \theta_{\mathrm{j}}$ for every pixel with coordinates $\mathrm{x}$, $y$ images of any pixels on the same line in the same accumulator cell. When the $\rho$ and the $\theta$ have the same amount, and if the accumulation in the accumulator cell which is greater than the specified threshold, the values are converted back to the coordinates $\mathrm{x}, \mathrm{y}$. This recognizes that the line is rated at point on the plane $\mathrm{x}, \mathrm{y}$. In the same way, the CHT can find a curve and a circle as given in equation (8)

$$
r^{2}=\left(x-C_{x}\right)^{2}+\left(y-C_{y}\right)^{2}
$$

When $C_{x}, C_{y}$ is the center of the circle along the axis $\mathrm{x}$ and $\mathrm{y}, \mathrm{r}$ is the radius of the circle or arc. In the case of a circle, accumulator cell is $\mathrm{A}(\mathrm{i}, \mathrm{j}) 3$-dimensional, the parameter will contain $C_{x}, C_{y}$ and $\mathrm{r}$ for calculate the coordinates $\mathrm{x}, \mathrm{y}$ on the same circle or arc. The method is used the same as a straight line.

\section{VASCULAR SYSTEM AND ARM}

The conveyor system simulation was designed using a servo motor driving the conveyor belt width $10 \mathrm{~cm}, 62 \mathrm{~cm}$ long, as shown in Fig. 7. The robot arm is a revolute robot type (RRR) and controlled by the microcontroller as shown in Fig. 8 and 9.

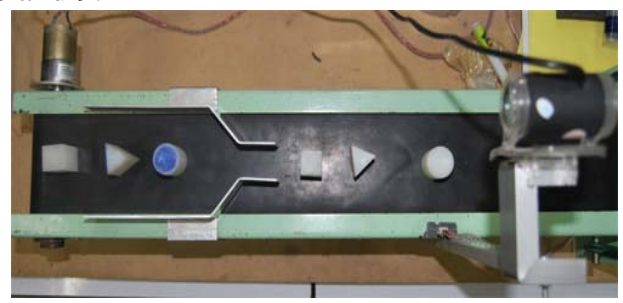

Fig. 7. Conveyor model

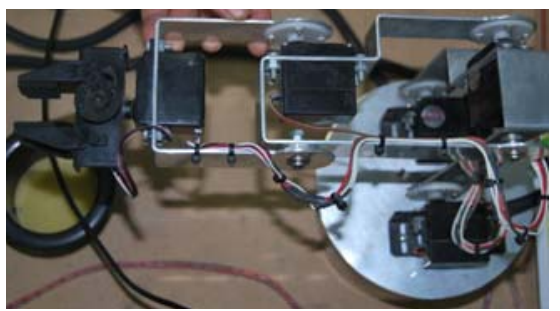

Fig. 8. A revolute robot arm

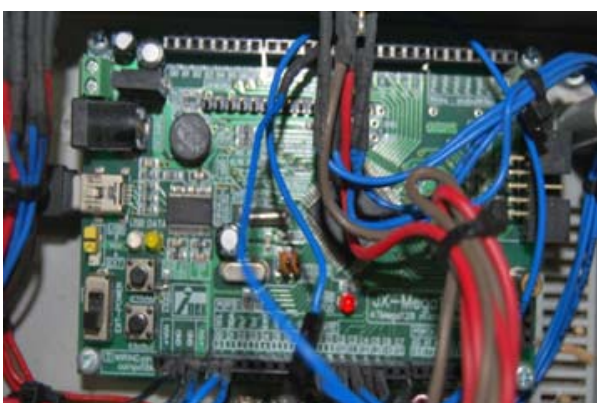

Fig. 9. Microcontroller for the control the robot arm

\section{EXPERIMENTS AND RESULT}

The aim of this study was to determine the efficiency of inspection triangle, squares, and circle objects by using the Hough Transform. The software was developed by using the Opencv library and Microsoft Visual $\mathrm{C}++$. The results are collected by testing three different objects. The experimental sequence was shown as follows.

\section{A. Preparation and Improvement of Image}

The first process is to import the images from webcam and improve the image as shown in Fig.10 (a)-(b).

\section{B. Object Edges Detection}

The object edges were detected by using sobel edge detection algorithm. The results are shown in Fig.10(c).
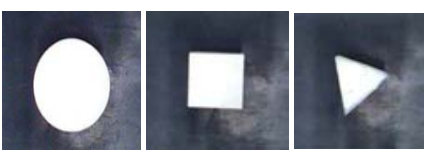

(a)
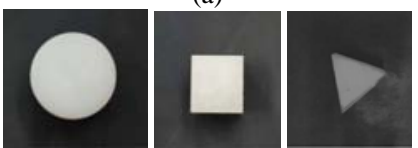

(b)
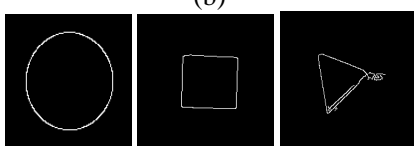

(c)

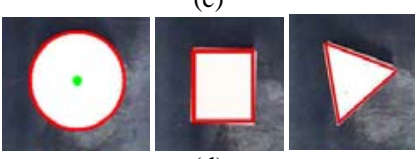

(d)

Fig. 10. Experiments of three object type (a) original image [RGB] (b) Through improved image [Gray level] (c) Sobel edge detection (d) Conventional hough transform

\section{Detect Objects}

The conventional Hough transform was used to detect the objects and the results are shown in Fig.10 (d) and Fig. 11.

\section{Separate Objects}

In this step, the robotic revolute robot arm was used to catch the object for separating. There are two experiments were implemented.

1) Each object type is implemented for robot to detect and separate 100 times. The result is shown in Table I.

2) Three object types are mixed for robot to detect and separate. This experiment consists of 100 pieces of each object type. The result is shown in Table II. 


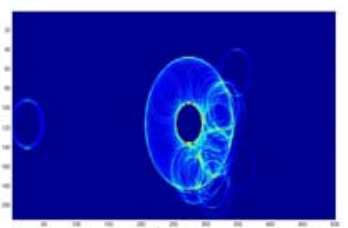

(a)

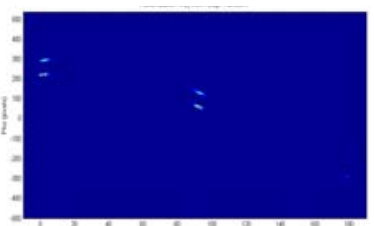

(b)

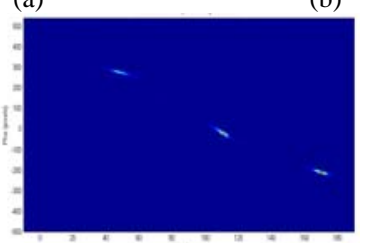

(c)

Fig. 11. Accumulation array from hough transform (a) Rigid circle shape (b) rectangular shape (c) triangle shape

TABLE I: PERCENTAGE OF DETECT AND SEPARATE THE SAmE OBJects

\begin{tabular}{llll}
\hline \hline Object & $\begin{array}{l}\text { Number of } \\
\text { objects }\end{array}$ & $\begin{array}{l}\text { Number of } \\
\text { objects } \\
\text { detected }\end{array}$ & $\begin{array}{l}\text { Error } \\
\text { percentage }\end{array}$ \\
\hline rigid circle & 100 & 98 & $2 \%$ \\
rectangular & 100 & 97 & $3 \%$ \\
triangular & 100 & 96 & $4 \%$ \\
\hline \hline
\end{tabular}

TABLE II : Percentage of Detect AND Separate the Three OBJect

\begin{tabular}{llll}
\hline \multicolumn{4}{c}{ TYPES } \\
\hline Object & $\begin{array}{l}\text { Number of } \\
\text { objects }\end{array}$ & $\begin{array}{l}\text { Number of } \\
\text { objects } \\
\text { detected }\end{array}$ & $\begin{array}{l}\text { Error } \\
\text { percentage }\end{array}$ \\
\hline rigid circle & 100 & 98 & $2 \%$ \\
rectangular & 100 & 96 & $4 \%$ \\
triangular & 100 & 96 & $4 \%$ \\
\hline \hline
\end{tabular}

\section{CONCLUSIONS}

The conventional Hough transform method can be used for the object identification and shape recognition. The algorithm can be implemented with the robot arm to separate the objects. The experimental results have an error is occurred form the light affect and the angle of the camera. However, the error maximum was $4 \%$.

\section{REFERENCES}

[1] B. Jayanta and K. P. Sankar, "Theoretical Quantification of Shape Distortion in Fuzzy Hough,” Fuzzy Sets and Systems, pp. 227-250.

[2] M. Subhransu and M. Jitendra, “Object Detection using a Max-Margin Hough Transform," presented at the IEEE Computer Society Conference on Computer Vision and Pattern Recognition, Fontainebleau Resort, Miami Beach, Florida, June 20-25, 2009.

[3] R. Mohamed, Y. Haniza, S. Puteh, M. S. A. Yeon, M. S. A. Rahman Saad, Y. Sazali, M. R. Mamat, and M. Karthigayan, “Object Detection using Circular Hough Transform,” American Journal of Applied Sciences, vol. 2, no. 12, pp. 1606-1609, 2005.

[4] S. Marcin and D. B. Ignacy, "Circular Object Detection Using a Modified Hough Transform,” Int. J. Appl. Math. Comput. Sci., vol. 18, no. 1, pp. 85-91, 2008.

[5] K. Lee, "Application of the Hough Transform," University of Massachusetts, Lowell, January, 2006.

[6] S. Kaset, S. Thirachat, and S. Sasi, "Hough Transform Modification," in Proc. The $29^{\text {th }}$ Kasetsart University Annual Conference, 2001, pp 209-216.

[7] K. Kourosh, "Extending Generalized Hough Transform to Detect 3D Objects in Laser Range Data,” presented at the ISPRS Workshop on Laser Scanning 2007 and SilviLaser 2007, Espoo, Finland, September 12-14, 2007.

[8] A. A. Abdallah, and A. A. Ayman, "Edge Detection in Digital Images Using Fuzzy Logic Technique,” presented at the World Academy of Science, Engineering and Technology, vol. 51, 2009.

[9] V. Mittal, “Edge Detection Technique Using Fuzzy Logic,” presented at the Thapar University, Patiala, May, 2008.

[10] G. Sudhavani and K. Sathyaprasad, "Segmentation of Lip Images by Modified Fuzzy C-means Clustering Algorithm,” IJCSNS International Journal of Computer Science and Network Security, vol. 9, no. 4, April, 2009.

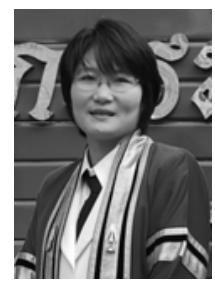

Jaruwan Toontham received her B.S.I.Ed. from Rajamungala University of Technology, Thailand, in 1994. She received her MS.Ed from the King Mongkut's University of Technology North Bangkok (KMUTNB), Thailand, in 1999. Now, she is a Ph.D student in King Mongkut's University of Technology North Bangkok (KMUTNB), Thailand.

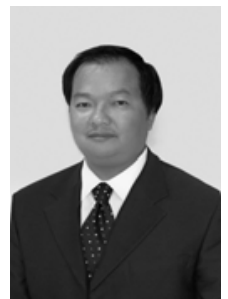

Chaiyapon Thongchaisuratkrul received his B.S.I.Ed. and M. Eng from the King Mongkut's University of Technology North Bangkok (KMUTNB), Thailand, in 1989 and 1992 respectively. He received his Ph.D. from the Vanderbilt University, USA in 2001. He joined KMUTNB as a lecture of Teacher Training in Electrical Engineering Department in 1989. Now he is an assistant professor in the department. He served as the vice president for network and conference of the Thai Robotics Society in 2008. His research interests include intelligent control systems, robotics, energy conservation, and energy resources. 